\title{
On ideal convergence Fibonacci difference sequence spaces
}

\author{
Vakeel A. Khan ${ }^{1 *}$, Rami K.A. Rababah², Kamal M.A.S. Alshlool' ${ }^{1}$, Sameera A.A. Abdullah and Ayaz Ahmad ${ }^{3}$
}

"Correspondence:

vakhanmaths@gmail.com

'Department of Mathematics,

Aligarh Muslim University, Aligarh, India

Full list of author information is available at the end of the article

\begin{abstract}
The Fibonacci sequence was firstly used in the theory of sequence spaces by Kara and Başarir (Casp. J. Math. Sci. 1(1):43-47, 2012). Afterward, Kara (J. Inequal. Appl. 2013(1):38, 2013) defined the Fibonacci difference matrix $\hat{F}$ by using the Fibonacci sequence $\left(f_{n}\right)$ for $n \in\{0,1, \ldots\}$ and introduced new sequence spaces related to the matrix domain of $\hat{F}$. In this paper, by using the Fibonacci difference matrix $\hat{F}$ defined by the Fibonacci sequence and the notion of ideal convergence, we introduce the Fibonacci difference sequence spaces $c_{0}^{\prime}(\hat{F}), C^{\prime}(\hat{F})$, and $\ell_{\infty}^{\prime}(\hat{F})$. Further, we study some inclusion relations concerning these spaces. In addition, we discuss some properties on these spaces such as monotonicity and solidity.
\end{abstract}

Keywords: Fibonacci difference matrix; Fibonacci /-convergence; Fibonacci I-Cauchy; Fibonacci I-bounded; Lipschitz function

\section{Introduction}

Let $\mathbb{N}$ and $\mathbb{R}$ denote the sets of natural and real numbers, respectively. By $\omega$ we denote the vector space of all real sequences. Any vector subspace of $\omega$ is called a sequence space. Throughout the paper, $\ell_{\infty}, c$, and $c_{0}$ are the classes of bounded, convergent, and null sequences, respectively, with norm $\|x\|_{\infty}=\sup _{k \in \mathbb{N}}\left|x_{k}\right|$. Let $\lambda$ and $\mu$ be two sequence spaces, and let $A=\left(a_{n k}\right)$ be an infinite matrix of real numbers $a_{n k}, n, k \in \mathbb{N}$. Then we say that $A$ defines a matrix transformation from $\lambda$ into $\mu$, and we denote it by writing $A: \lambda \longrightarrow \mu$ if for every sequence $x=\left(x_{k}\right) \in \lambda$, the sequence $A x=\left\{A_{n}(x)\right\}$, the $A$-transform of $x$, is in $\mu$, where

$$
A_{n}(x)=\sum_{k=0}^{\infty} a_{n k} x_{k} \quad \text { for } n \in \mathbb{N} .
$$

$\operatorname{By}(\lambda, \mu)$ we denote the class of all matrices $A$. Thus $A \in(\lambda, \mu)$ if and only if the series on the right side of (1.1) converges for each $n \in \mathbb{N}$ and every $x \in \lambda$ and $A x \in \mu$ for all $x \in \lambda$, where $A_{n}=\left(a_{n k}\right)_{k \in \mathbb{N}}$ denotes the sequence in the $n$th row of $A$. The concept of matrix domain has fundamental importance for this study. So, the matrix domain of an infinite matrix $A$ in a sequence space $\lambda$ is defined by

$$
\lambda_{A}:=\left\{x=\left(x_{k}\right) \in \omega: A x \in \lambda\right\},
$$

(c) The Author(s) 2018. This article is distributed under the terms of the Creative Commons Attribution 4.0 International License (http://creativecommons.org/licenses/by/4.0/), which permits unrestricted use, distribution, and reproduction in any medium, provided you give appropriate credit to the original author(s) and the source, provide a link to the Creative Commons license, and indicate if changes were made. 
which is a sequence space. If $A=\Delta$, where $\triangle$ is the backward difference matrix defined by

$$
\Delta=\Delta_{n k}= \begin{cases}(-1)^{n-k}, & n-1 \leq k \leq n, \\ 0, & 0 \leq k<n-1 \text { or } k>n,\end{cases}
$$

for all $n, k \in \mathbb{N}$, then $\lambda_{\Delta}$ is called the difference sequence space defined by the domain of a triangle matrix $A$ whenever $\lambda$ is a normed linear space or paranormed sequence space. The notion of difference sequence spaces was introduced by Kizmaz [22] as follows:

$$
\lambda(\triangle):=\left\{x=\left(x_{n}\right) \in \omega:\left(x_{n}-x_{n+1}\right) \in \lambda\right\}
$$

for $\lambda \in\left\{\ell_{\infty}, c, c_{0}\right\}$. In recent years, some researchers have addressed the approach to constructing a new sequence space by means of the matrix domain of a particular limitation method; see, for instance, $[2-4,10,11,15,20,26]$ and the references therein. Quite recently, Kara [12] has introduced the difference sequence space

$$
\ell_{\infty}(\hat{F})=\left\{x=\left(x_{n}\right) \in \omega: \sup _{n \in \mathbb{N}}\left|\frac{f_{n}}{f_{n+1}} x_{n}-\frac{f_{n+1}}{f_{n}} x_{n-1}\right|<\infty\right\},
$$

which is derived by the Fibonacci difference matrix $\hat{F}=\left(\hat{f}_{n k}\right)$ defined as follows:

$$
\hat{f}_{n k}= \begin{cases}-\frac{f_{n+1}}{f_{n}}, & k=n-1, \\ \frac{f_{n}}{f_{n+1}}, & k=n, \\ 0, & 0 \leq k<n-1 \text { or } k>n,\end{cases}
$$

for all $n, k \in \mathbb{N}$, where $\left\{f_{n}\right\}_{n=0}^{\infty}$ is the sequence of Fibonacci numbers defined by the linear recurrence equalities $f_{0}=f_{1}=1$ and $f_{n}=f_{n-1}+f_{n-2}, n \geq 2$, with the following fundamental properties (see Koshy [23]):

$$
\begin{aligned}
& \lim _{n \rightarrow \infty} \frac{f_{n+1}}{f_{n}}=\frac{1+\sqrt{5}}{2}=\alpha \quad \text { (Golden Ratio), } \\
& \sum_{k=0}^{n} f_{k}=f_{n+2}-1 \quad(n \in \mathbb{N}) \\
& \sum_{k} \frac{1}{f_{k}} \text { converges, } \\
& f_{n-1} f_{n+1}-f_{n}^{2}=(-1)^{n+1}, \quad n \geq 1 \text { (Cassini's formula), }
\end{aligned}
$$

which yields $f_{n-1}^{2}+f_{n} f_{n-1}-f_{n}^{2}=(-1)^{n+1}$ by substituting for $f_{n+1}$ in Cassini's formula.

For a more detailed information about Fibonacci sequence spaces, we refer to [5-7, 18, $25]$. By using the same infinite Fibonacci matrix $\hat{F}$ and the same technique, Başarir et al. [1] have introduced the Fibonacci difference sequence spaces $c_{0}(\hat{F})$ and $c(\hat{F})$ as the sets of all sequences whose $\hat{F}$-transforms are in the spaces $c_{0}$ and $c$, respectively, that is,

$$
c_{0}(\hat{F}):=\left\{x=\left(x_{n}\right) \in \omega: \lim _{n \rightarrow \infty} \hat{F}_{n}(x)=0\right\}
$$


and

$$
c(\hat{F}):=\left\{x=\left(x_{n}\right) \in \omega: \exists \ell \in \mathbb{R} \ni \lim _{n \rightarrow \infty} \hat{F}_{n}(x)=\ell\right\},
$$

where the sequence $\hat{F}_{n}(x)$ is the $\hat{F}$-transform of a sequence $x=\left(x_{n}\right) \in \omega$, defined as follows:

$$
\hat{F}_{n}(x)= \begin{cases}\frac{f_{0}}{f_{1}} x_{0}=x_{0}, & n=0, \\ \frac{f_{n}}{f_{n+1}} x_{n}-\frac{f_{n+1}}{f_{n}} x_{n-1}, & n \geq 1 .\end{cases}
$$

By an ideal we mean a family of sets $I \subset P(X)$ (where $P(X)$ is the power set of $X$ ) such that (i) $\emptyset \in I$, (ii) $A \cup B \in I$ for all $A, B \in I$, and (iii) for each $A \in I$ and $B \subset A$, we have $B \in I$; $I$ is called admissible in $X$ if it contains all singletons, that is, if $I \supset\{\{x\}: x \in X\}$. A filter on $X$ is a nonempty family of sets $\mathcal{F} \subset P(X)$ satisfying (i) $\emptyset \notin \mathcal{F}$, (ii) $A, B \in \mathcal{F}$ implies that $A \cap B \in \mathcal{F}$, and (iii) for any $A \in \mathcal{F}$ and $B \supset A$, we have $B \in \mathcal{F}$. For each ideal $I$, there is a filter $\mathcal{F}(I)$ corresponding to $I$ (a filter associated with ideal $I$ ), that is, $\mathcal{F}(I)=\left\{K \subseteq X: K^{c} \in\right.$ $I\}$, where $K^{c}=X \backslash K$. In 1999, Kostyrko et al. [24] defined the notion of $I$-convergence, which depends on the structure of ideals of subsets of $\mathbb{N}$ as a generalization of statistical convergence introduced by Fast [9] and Steinhaus [29] in 1951. Later on, the notion of I-convergence was further investigated from the sequence space point of view and linked with the summability theory by Šalát et al. [27], Tripathy and Hazarika [30-32], Khan and Ebadullah [19], Das et al. [8], and many other authors. Šalát et al. [28] extended the notion of summability fields of an infinite matrix of operators $A$ with the help of the notion of $I$-convergence, that is, the notion of $I$-summability and introduced new sequence spaces $c_{A}^{I}$ and $m_{A}^{I}$, the $I$-convergence field and bounded $I$-convergence field of an infinite matrix $A$, respectively. For further details on ideal convergence, we refer to [14, 16, 17].

Throughout the paper, $c_{0}^{I}, c^{I}$, and $\ell_{\infty}^{I}$ denote the $I$-null, $I$-convergent, and $I$-bounded sequence spaces, respectively. In this paper, by combining the definitions of Fibonacci difference matrix $\hat{F}$ and ideal convergence we introduce the sequence spaces $c_{0}^{I}(\hat{F}), c^{I}(\hat{F})$, and $\ell_{\infty}^{I}(\hat{F})$. Further, we study some topological and algebraic properties of these spaces. Also, we study some inclusion relations concerning these spaces.

Now, we recall some definitions and lemmas, which will be used throughout the paper.

Definition 1.1 $([9,29])$ A sequence $x=\left(x_{n}\right) \in \omega$ is said to be statistically convergent to a number $\ell \in \mathbb{R}$ if, for every $\epsilon>0$, the natural density of the set $\left\{n \in \mathbb{N}:\left|x_{n}-\ell\right| \geq \epsilon\right\}$ equals zero, and we write $s t-\lim x_{n}=\ell$. If $\ell=0$, then $x=\left(x_{n}\right) \in \omega$ is said to be st-null.

Definition 1.2 ([27]) A sequence $x=\left(x_{n}\right) \in \omega$ is said to be $I$-Cauchy if, for every $\epsilon>0$, there exists a number $N=N(\epsilon)$ such that the set $\left\{n \in \mathbb{N}:\left|x_{n}-x_{N}\right| \geq \epsilon\right\} \in I$.

Definition 1.3 ([24]) A sequence $x=\left(x_{n}\right) \in \omega$ is said to be $I$-convergent to a number $\ell \in \mathbb{R}$ if, for every $\epsilon>0$, the set $\left\{n \in \mathbb{N}:\left|x_{n}-\ell\right| \geq \epsilon\right\} \in I$, and we write $I-\lim x_{n}=\ell$. If $\ell=0$, then $\left(x_{n}\right) \in \omega$ is said to be $I$-null.

Definition 1.4 ([19]) A sequence $x=\left(x_{n}\right) \in \omega$ is said to be $I$-bounded if there exists $K>0$ such that the set $\left\{n \in \mathbb{N}:\left|x_{n}\right| \geq K\right\} \in I$. 
Definition 1.5 ([27]) Let $x=\left(x_{n}\right)$ and $z=\left(z_{n}\right)$ be two sequences. We say that $x_{n}=z_{n}$ for almost all $n$ relative to $I$ (in short, a.a.n.r.I) if the set $\left\{n \in \mathbb{N}: x_{n} \neq z_{n}\right\} \in I$.

Definition 1.6 ([27]) A sequence space $E$ is said to be solid or normal if $\left(\alpha_{n} x_{n}\right) \in E$ for any sequence $\left(x_{n}\right) \in E$ and any sequence of scalars $\left(\alpha_{n}\right) \in \omega$ with $\left|\alpha_{n}\right|<1$ for all $n \in \mathbb{N}$.

Lemma 1.1 ([27]) Every solid space is monotone.

Definition 1.7 ([27]) A sequence space $E$ is said to be a sequence algebra if $\left(x_{n}\right) *\left(z_{n}\right)=$ $\left(x_{n} \cdot z_{n}\right) \in E$ for all $\left(x_{n}\right),\left(z_{n}\right) \in E$.

Definition 1.8 ([27]) Let $K=\left\{n_{i} \in \mathbb{N}: n_{1}<n_{2}<\cdots\right\} \subseteq \mathbb{N}$, and let $E$ be a sequence space. The $K$-step space of $E$ is the sequence space

$$
\lambda_{K}^{E}=\left\{\left(x_{n_{i}}\right) \in \omega:\left(x_{n}\right) \in E\right\} .
$$

A canonical preimage of a sequence $\left(x_{n_{i}}\right) \in \lambda_{K}^{E}$ is the sequence $\left(y_{n}\right) \in \omega$ defined as

$$
y_{n}= \begin{cases}x_{n} & \text { if } n \in K \\ 0 & \text { otherwise }\end{cases}
$$

A canonical preimage of the step space $\lambda_{K}^{E}$ is the set of canonical preimages of all elements in $\lambda_{K}^{E}$, that is, $y$ is in the canonical preimage of $\lambda_{K}^{E}$ iff $y$ is the canonical preimage of some element $x \in \lambda_{K}^{E}$.

Definition 1.9 ([27]) A sequence space $E$ is said to be monotone if it contains the canonical preimages of its step space (i.e., if for all infinite $K \subseteq \mathbb{N}$ and $\left(x_{n}\right) \in E$, the sequence $\left(\alpha_{n} x_{n}\right)$ with $\alpha_{n}=1$ for $n \in K$ and $\alpha_{n}=0$ otherwise belongs to $E$ ).

Definition 1.10 A map $h$ defined on a domain $D \subset X$ (i.e., $h: D \subset X \rightarrow \mathbb{R}$ ) is said to satisfy the Lipschitz condition if $|h(x)-h(y)| \leq K|x-y|$, where $K$ is called the Lipschitz constant.

Remark 1.1 ([27]) The convergence field of $I$-convergence is the set

$$
\mathcal{F}(I)=\left\{x=\left(x_{k}\right) \in \ell_{\infty}: \text { there exists } I-\lim x \in \mathbb{R}\right\} .
$$

Definition 1.11 ([24]) The convergence field $\mathcal{F}(I)$ is a closed linear subspace of $\ell_{\infty}$ with respect to the supremum norm, $\mathcal{F}(I)=\ell_{\infty} \cap c^{I}$.

Lemma 1.2 ([28]) Let $K \in \mathcal{F}(I)$ and $M \subseteq \mathbb{N}$. If $M \notin I$, then $M \cap K \notin I$.

Definition 1.12 ([27]) The function $h: D \subset X \longrightarrow \mathbb{R}$ defined by $h(x)=I-\lim x$ for all $x \in$ $\mathcal{F}(I)$ is a Lipschitz function. 


\section{I-Convergence Fibonacci difference sequence spaces}

In this section, we introduce the sequence spaces as the sets of sequences whose $\hat{F}$ transforms are in the spaces $c_{0}^{I}, c^{I}$, and $\ell_{\infty}^{I}$. Further, we present some inclusion theorems and study some topological and algebraic properties on these resulting. Throughout the paper, we suppose that a sequence $x=\left(x_{n}\right) \in \omega$ and $\hat{F}_{n}(x)$ are connected by relation (1.5) and $I$ is an admissible ideal of subset of $\mathbb{N}$. We define

$$
\begin{aligned}
& c_{0}^{I}(\hat{F}):=\left\{x=\left(x_{n}\right) \in \omega:\left\{n \in \mathbb{N}:\left|\hat{F}_{n}(x)\right| \geq \epsilon\right\} \in I\right\}, \\
& c^{I}(\hat{F}):=\left\{x=\left(x_{n}\right) \in \omega:\left\{n \in \mathbb{N}:\left|\hat{F}_{n}(x)-L\right| \geq \epsilon \text { for some } L \in \mathbb{R}\right\} \in I\right\}, \\
& \ell_{\infty}^{I}(\hat{F}):=\left\{x=\left(x_{n}\right) \in \omega: \exists K>0 \text { s.t. }\left\{n \in \mathbb{N}:\left|\hat{F}_{n}(x)\right| \geq K\right\} \in I\right\},
\end{aligned}
$$

We write

$$
m_{0}^{I}(\hat{F}):=c_{0}^{I}(\hat{F}) \cap \ell_{\infty}(\hat{F})
$$

and

$$
m^{I}(\hat{F}):=c^{I}(\hat{F}) \cap \ell_{\infty}(\hat{F})
$$

With notation (1.2), the spaces $c_{0}^{I}(\hat{F}), c^{I}(\hat{F}), \ell_{\infty}^{I}(\hat{F}), m^{I}(\hat{F})$, and $m_{0}^{I}(\hat{F})$ can be redefined as follows:

$$
\begin{aligned}
& c_{0}^{I}(\hat{F})=\left(c_{0}^{I}\right)_{\hat{F}}, \quad c^{I}(\hat{F})=\left(c^{I}\right)_{\hat{F}}, \quad \ell_{\infty}^{I}(\hat{F})=\left(\ell_{\infty}^{I}\right)_{\hat{F}}, \\
& m^{I}(\hat{F})=\left(m^{I}\right)_{\hat{F}}, \quad \text { and } \quad m_{0}^{I}(\hat{F})=\left(m_{0}^{I}\right)_{\hat{F}} .
\end{aligned}
$$

Definition 2.1 Let $I$ be an admissible ideal of subsets of $\mathbb{N}$. A sequence $x=\left(x_{n}\right) \in \omega$ is called Fibonacci $I$-Cauchy if for each $\epsilon>0$, there exists a number $N=N(\epsilon) \in \mathbb{N}$ such that $\left\{n \in \mathbb{N}:\left|\hat{F}_{n}(x)-\hat{F}_{N}(x)\right| \geq \epsilon\right\} \in I$.

Example 2.1 Define $I_{f}=\{A \subseteq \mathbb{N}: A$ is finite $\}$. Then $I_{f}$ is an admissible ideal in $\mathbb{N}$, and $c^{I_{f}}(\hat{F})=c(\hat{F})$.

Example 2.2 Define the nontrivial ideal $I_{d}=\{A \subseteq \mathbb{N}: d(A)=0\}$, where $d(A)$ is the natural density of a set $A$. In this case, $c^{I_{d}}(\hat{F})=S(\hat{F})$, where $S(\hat{F})$ is the space of Fibonacci difference statistically convergent sequence defined as

$$
S(\hat{F}):=\left\{x=\left(x_{n}\right) \in \omega: d\left(\left\{n \in \mathbb{N}:\left|\hat{F}_{n}(x)-L\right| \geq \epsilon\right\}\right)=0 \text { for some } L \in \mathbb{R}\right\}
$$

Theorem 2.1 The sequence spaces $c^{I}(\hat{F}), c_{0}^{I}(\hat{F}), \ell_{\infty}^{I}(\hat{F}), m_{0}^{I}(\hat{F})$, and $m^{I}(\hat{F})$ are linear over $\mathbb{R}$.

Proof Let $x=\left(x_{n}\right)$ and $y=\left(y_{n}\right)$ be two arbitrary elements of the space $c^{I}(\hat{F})$, and let $\alpha, \beta$ are scalars. Then, for given $\epsilon>0$, there exist $L_{1}, L_{2} \in \mathbb{R}$ such that

$$
\left\{n \in \mathbb{N}:\left|\hat{F}_{n}(x)-L_{1}\right| \geq \frac{\epsilon}{2}\right\} \in I
$$


and

$$
\left\{n \in \mathbb{N}:\left|\hat{F}_{n}(y)-L_{2}\right| \geq \frac{\epsilon}{2}\right\} \in I \text {. }
$$

Now, let

$$
A_{1}=\left\{n \in \mathbb{N}:\left|\hat{F}_{n}(x)-L_{1}\right|<\frac{\epsilon}{2|\alpha|}\right\} \in \mathcal{F}(I)
$$

and

$$
A_{2}=\left\{n \in \mathbb{N}:\left|\hat{F}_{n}(y)-L_{2}\right|<\frac{\epsilon}{2|\beta|}\right\} \in \mathcal{F}(I)
$$

be such that $A_{1}^{c}, A_{2}^{c} \in I$. Then

$$
\begin{aligned}
A_{3} & =\left\{n \in \mathbb{N}:\left|\alpha \hat{F}_{n}(x)+\beta \hat{F}_{n}(y)-\left(\alpha L_{1}+\beta L_{2}\right)\right|<\epsilon\right\} \\
& \supseteq\left\{\left\{n \in \mathbb{N}:\left|\hat{F}_{n}(x)-L_{1}\right|<\frac{\epsilon}{2|\alpha|}\right\} \cap\left\{n \in \mathbb{N}:\left|\hat{F}_{n}(y)-L_{2}\right|<\frac{\epsilon}{2|\beta|}\right\}\right\} .
\end{aligned}
$$

Thus, the sets on the right-hand side of (2.7) belong to $\mathcal{F}(I)$. By the definition of the filter associated with an ideal the complement of the set on the left-hand side of (2.7) belongs to $I$. This implies that $(\alpha x+\beta y) \in c^{I}(\hat{F})$. Hence $c^{I}(\hat{F})$ is a linear space. The proof of the remaining results is similar.

Theorem 2.2 The spaces $X(\hat{F})$ are normed spaces with the norm

$$
\|x\|_{X(\hat{F})}=\sup _{n}\left|\hat{F}_{n}(x)\right|, \quad \text { where } X \in\left\{m^{I}, m_{0}^{I}\right\} .
$$

Proof The proof of the result is easy by existing techniques and hence is omitted.

Theorem 2.3 Let $I \subseteq 2^{\mathbb{N}}$ be a nontrivial ideal. Then the inclusion $c(\hat{F}) \subset c^{I}(\hat{F})$ is strict.

Proof We know that $c \subseteq c^{I}$ and, for any $X$ and $Y$ spaces, $X \subseteq Y$ implies $X(\hat{F}) \subseteq Y(\hat{F})$ (see [21], Lemma 2.1). Hence it is easy to see that $c(\hat{F}) \subset c^{I}(\hat{F})$. The following example shows the strictness of the inclusion.

Example 2.3 Define the sequence $x=\left(x_{n}\right) \in \omega$ such that

$$
\hat{F}_{n}(x)= \begin{cases}\sqrt{n} & \text { if } n \text { is a square } \\ 0 & \text { otherwise. }\end{cases}
$$

Then $x \in c^{I_{d}}(\hat{F})$, but $x \notin c(\hat{F})$.

Example 2.4 Define the ideal $I$ such that

$A \in I \quad \Longleftrightarrow \quad A$ eventually contains only even natural numbers. 
Then $I$ is a nontrivial ideal in $\mathbb{N}$. When

$$
\hat{F}_{n}(x)=(1,1,1,0,1,1,1,0,0,1,0,1,0, \ldots)
$$

we have

$$
A_{\epsilon}=\left\{n \in \mathbb{N}: \hat{F}_{n}(x) \neq 0\right\}=\{1,2,3,5,6,7,10,12,14,16,18, \ldots\}
$$

and $\left(x_{n}\right) \in c_{0}^{I}(\hat{F})$. Hence $A_{\epsilon} \in I$ and $\hat{F}_{n}(x) \in c^{I}$. Now let us look at the statistical convergence of the sequence:

$$
\lim _{n \rightarrow \infty} \frac{1}{n}\left|A_{\epsilon}\right|=\lim _{n \rightarrow \infty} \frac{1}{n}\left|B+\frac{n}{2}\right|=\frac{1}{2},
$$

where $B$ is a finite number, and $\left|A_{\epsilon}\right|$ is the cardinality of $A_{\epsilon}$. Hence $\hat{F}_{n}(x) \notin S$.

Theorem 2.4 A sequence $x=\left(x_{n}\right) \in \omega$ Fibonacci I-converges if and only if for every $\epsilon>0$, there exists $N=N(\epsilon) \in \mathbb{N}$ such that

$$
\left\{n \in \mathbb{N}:\left|\hat{F}_{n}(x)-\hat{F}_{N}(x)\right|<\epsilon\right\} \in \mathcal{F}(I)
$$

Proof Suppose that a sequence $x=\left(x_{n}\right) \in \omega$ is Fibonacci $I$-convergent to some number $L \in \mathbb{R}$. Then, for given $\epsilon>0$, the set

$$
B_{\epsilon}=\left\{n \in \mathbb{N}:\left|\hat{F}_{n}(x)-L\right|<\frac{\epsilon}{2}\right\} \in \mathcal{F}(I)
$$

Fix an integer $N=N(\epsilon) \in B_{\epsilon}$. Then we have

$$
\left|\hat{F}_{n}(x)-\hat{F}_{N}(x)\right| \leq\left|\hat{F}_{n}(x)-L\right|+\left|L-\hat{F}_{N}(x)\right|<\frac{\epsilon}{2}+\frac{\epsilon}{2}=\epsilon
$$

for all $n \in B_{\epsilon}$. Hence (2.9) holds.

Conversely, suppose that (2.9) holds for all $\epsilon>0$. Then

$$
C_{\epsilon}=\left\{n \in \mathbb{N}: \hat{F}_{n}(x) \in\left[\hat{F}_{n}(x)-\epsilon, \hat{F}_{n}(x)+\epsilon\right]\right\} \in \mathcal{F}(I) \quad \text { for all } \epsilon>0 .
$$

Let $J_{\epsilon}=\left[\hat{F}_{n}(x)-\epsilon, \hat{F}_{n}(x)+\epsilon\right]$. Fixing $\epsilon>0$, we have $C_{\epsilon} \in \mathcal{F}(I)$ and $C_{\frac{\epsilon}{2}} \in \mathcal{F}(I)$. Hence $C_{\epsilon} \cap$ $C_{\frac{\epsilon}{2}} \in \mathcal{F}(I)$. This implies that

$$
J=J_{\epsilon} \cap J_{\frac{\epsilon}{2}} \neq \emptyset
$$

that is,

$$
\left\{n \in \mathbb{N}: \hat{F}_{n}(x) \in J\right\} \in \mathcal{F}(I)
$$

and thus

$$
\operatorname{diam}(J) \leq \frac{1}{2} \operatorname{diam}\left(J_{\epsilon}\right)
$$


where $\operatorname{diam}(J)$ denotes the length of an interval $J$. Proceeding in this way, by induction we get a sequence of closed intervals

$$
J_{\epsilon}=I_{0} \supseteq I_{1} \supseteq \cdots \supseteq I_{n} \supseteq \cdots
$$

such that

$$
\operatorname{diam}\left(I_{n}\right) \leq \frac{1}{2} \operatorname{diam}\left(I_{n-1}\right) \quad \text { for } n=2,3, \ldots
$$

and

$$
\left\{n \in \mathbb{N}: \hat{F}_{n}(x) \in I_{n}\right\} \in \mathcal{F}(I)
$$

Then there exists a number $L \in \bigcap_{n \in \mathbb{N}} I_{n}$, and it is a routine work to verify that $L=$ $I-\lim \hat{F}_{n}(x)$, showing that $x=\left(x_{n}\right) \in \omega$ Fibonacci $I$-converges. Hence the result.

Theorem 2.5 Let I be an admissible ideal. Then the following are equivalent:

(a) $\left(x_{n}\right) \in c^{I}(\hat{F})$;

(b) There exists $\left(y_{n}\right) \in c(\hat{F})$ such that $x_{n}=y_{n}$ for a.a.n.r.I;

(c) There exist $\left(y_{n}\right) \in c(\hat{F})$ and $\left(z_{n}\right) \in c_{0}^{I}(\hat{F})$ such that $x_{n}=y_{n}+z_{n}$ for all $n \in \mathbb{N}$ and $\left\{n \in \mathbb{N}:\left|\hat{F}_{n}(x)-L\right| \geq \epsilon\right\} \in I$

(d) There exists a subset $K=\left\{n_{i}: i \in \mathbb{N}, n_{1}<n_{2}<n_{3}<\cdots\right\}$ of $\mathbb{N}$ such that $K \in \mathcal{F}(I)$ and $\lim _{n \rightarrow \infty}\left|\hat{F}_{n_{i}}(x)-L\right|=0$.

Proof (a) implies (b). Let $x=\left(x_{n}\right) \in c^{I}(\hat{F})$. Then, for any $\epsilon>0$, there exists $L \in \mathbb{R}$ such that

$$
\left\{n \in \mathbb{N}:\left|\hat{F}_{n}(x)-L\right| \geq \epsilon\right\} \in I
$$

Let $\left(m_{t}\right)$ be an increasing sequence with $m_{t} \in \mathbb{N}$ such that

$$
\left\{n \leq m_{t}:\left|\hat{F}_{n}(x)-L\right| \geq t^{-1}\right\} \in I \text {. }
$$

Define the sequence $y=\left(y_{n}\right)$ as $y_{n}=z_{n}$ for all $n \leq m_{1}$ and, for $m_{t}<n<m_{t+1}, t \in \mathbb{N}$, as

$$
y_{n}= \begin{cases}x_{n} & \text { if }\left|\hat{F}_{n}(x)-L\right|<t^{-1} \\ L & \text { otherwise }\end{cases}
$$

Then $y_{n} \in c(\hat{F})$, and from the inclusion

$$
\left\{n \leq m_{t}: x_{n} \neq y_{n}\right\} \subseteq\left\{n \in \mathbb{N}:\left|\hat{F}_{n}(x)-L\right| \geq \epsilon\right\} \in I
$$

we get $x_{n}=y_{n}$ for a.a.n.r.I.

(b) implies (c). For $x=\left(x_{n}\right) \in c^{I}(\hat{F})$, there exists $y=\left(y_{n}\right) \in c(\hat{F})$ such that $x_{n}=y_{n}$ for a.a.n.r.I. Let $K=\left\{n \in \mathbb{N}: x_{n} \neq y_{n}\right\}$. Then $K \in I$. Define the sequence $z=\left(z_{n}\right)$ as

$$
z_{n}= \begin{cases}x_{n}-y_{n} & \text { if } n \in K, \\ 0 & \text { otherwise }\end{cases}
$$

Then $\left(z_{n}\right) \in c_{0}^{I}(\hat{F})$ and $\left(y_{n}\right) \in c(\hat{F})$. 
(c) implies (d). Let $P=\left\{n \in \mathbb{N}:\left|\hat{F}_{n}(x)\right| \geq \epsilon\right\} \in I$ and

$$
K=P^{c}=\left\{n_{i} \in \mathbb{N}: i \in \mathbb{N}, n_{1}<n_{2}<n_{3}<\cdots\right\} \in \mathcal{F}(I) .
$$

Then we have

$$
\lim _{i \rightarrow \infty}\left|\hat{F}_{n_{i}}(x)-L\right|=0
$$

(d) implies (a). Let $\epsilon>0$ be given and suppose that (c) holds. Then, for any $\epsilon>0$, by Lemma 1.2 we have

$$
\left\{n \in \mathbb{N}:\left|\hat{F}_{n}(x)-L\right| \geq \epsilon\right\} \subseteq K^{c} \cup\left\{n \in K:\left|\hat{F}_{n}(x)-L\right| \geq \epsilon\right\}
$$

Thus $\left(x_{n}\right) \in c^{I}(\hat{F})$.

Theorem 2.6 The inclusions $c_{0}^{I}(\hat{F}) \subset c^{I}(\hat{F}) \subset \ell_{\infty}^{I}(\hat{F})$ are strict.

Proof The inclusion $c_{0}^{I}(\hat{F}) \subset c^{I}(\hat{F})$ is obvious. Now, to show its strictness, consider the sequence $x=\left(x_{n}\right) \in \omega$ such that $\hat{F}_{n}(x)=1$. It easy to see that $\hat{F}_{n}(x) \in c^{I}$ but $\hat{F}_{n}(x) \notin c_{0}^{I}$, that is, $x \in c^{I}(\hat{F}) \backslash c_{0}^{I}(\hat{F})$. Next, let $x=\left(x_{n}\right) \in c^{I}(\hat{F})$. Then there exists $L \in \mathbb{R}$ such that $I-\lim \mid \hat{F}_{n}(x)-$ $L \mid=0$, that is,

$$
\left\{n \in \mathbb{N}:\left|\hat{F}_{n}(x)-L\right| \geq \epsilon\right\} \in I
$$

We have

$$
\left|\hat{F}_{n}(x)\right|=\left|\hat{F}_{n}(x)-L+L\right| \leq\left|\hat{F}_{n}(x)-L\right|+|L|
$$

From this it easily follows that the sequence $\left(x_{n}\right)$ must belong to $\ell_{\infty}^{I}(\hat{F})$. Further, we show the strictness of the inclusion $c^{I}(\hat{F}) \subset \ell_{\infty}^{I}(\hat{F})$ by constructing the following example.

Example 2.5 Consider the sequence $x=\left(x_{n}\right) \in \omega$ such that

$$
\hat{F}_{n}(x)= \begin{cases}\sqrt{n} & \text { if } n \text { is a square } \\ 1 & \text { if } n \text { is odd nonsquare } \\ 0 & \text { if } n \text { is even nonsquare }\end{cases}
$$

Then $\hat{F}_{n}(x) \in \ell_{\infty}^{I}$, but $\hat{F}_{n}(x) \notin c^{I}$, which implies that $x \in \ell_{\infty}^{I}(\hat{F}) \backslash c^{I}(\hat{F})$.

Thus the inclusion $c_{0}^{I}(\hat{F}) \subset c^{I}(\hat{F}) \subset \ell_{\infty}^{I}(\hat{F})$ is strict.

Remark 2.1 A Fibonacci bounded sequence is obviously Fibonacci $I$-bounded as the empty set belongs to the ideal $I$. However, the converse is not true. For example, consider the sequence

$$
\hat{F}_{n}(x)= \begin{cases}n & \text { if } n \text { is a square } \\ 0 & \text { otherwise }\end{cases}
$$


Clearly, $\hat{F}_{n}(x)$ is not a bounded sequence. However, $\left\{n \in \mathbb{N}:\left|\hat{F}_{n}(x)\right| \geq \frac{1}{2}\right\} \in I$. Hence $x=\left(x_{n}\right)$ is Fibonacci $I$-bounded.

Theorem 2.7 The spaces $m^{I}(\hat{F})$ and $m_{0}^{I}(\hat{F})$ are Banach spaces normed by $(2.8)$.

Proof Let $\left(x_{n}^{(i)}\right)$ be a Cauchy sequence in $m^{I}(\hat{F}) \subset \ell_{\infty}(\hat{F})$. Then $\left(x_{n}^{(i)}\right)$ converges in $\ell_{\infty}(\hat{F})$, and $\lim _{i \rightarrow \infty} \hat{F}_{n}^{(i)}(x)=\hat{F}_{n}(x)$. Let $I-\lim \hat{F}_{n}^{(j)}(x)=L_{i}$ for $i \in \mathbb{N}$. Then we have to show that

(i) $\left(L_{i}\right)$ is convergent say to $L$ and

(ii) $I-\lim \hat{F}_{n}(x)=L$.

(i) Since $\left(x_{n}^{(i)}\right)$ is a Cauchy sequence, for each $\epsilon>0$, there exists $n_{0} \in \mathbb{N}$ such that

$$
\left|\hat{F}_{n}^{(i)}(x)-\hat{F}_{n}^{(j)}(x)\right|<\frac{\epsilon}{3} \quad \text { for all } i, j \geq n_{0}
$$

Now let $E_{i}$ and $E_{j}$ be the following sets in $I$ :

$$
E_{i}=\left\{n \in \mathbb{N}:\left|\hat{F}_{n}^{(i)}(x)-L_{i}\right| \geq \frac{\epsilon}{3}\right\}
$$

and

$$
E_{j}=\left\{n \in \mathbb{N}:\left|\hat{F}_{n}^{(j)}(x)-L_{j}\right| \geq \frac{\epsilon}{3}\right\}
$$

Consider $i, j \geq n_{0}$ and $n \notin E_{i} \cap E_{j}$. Then we have

$$
\begin{aligned}
\left|L_{i}-L_{j}\right| & \leq\left|\hat{F}_{n}^{(i)}(x)-L_{i}\right|+\left|\hat{F}_{n}^{(j)}(x)-L_{j}\right|+\left|\hat{F}_{n}^{(i)}(x)-\hat{F}_{n}^{(j)}(x)\right| \\
& <\epsilon \quad \text { by (2.10), (2.11), and (2.12). }
\end{aligned}
$$

Thus $\left(L_{i}\right)$ is a Cauchy sequence in $\mathbb{R}$ and thus converges, say to $L$, that is, $\lim _{i \rightarrow \infty} L_{i}=L$.

(ii) Let $\delta>0$ be given. Then we can find $m_{0}$ such that

$$
\left|L_{i}-L\right|<\frac{\delta}{3} \quad \text { for each } i>m_{0}
$$

We have $\left(x_{n}^{(i)}\right) \rightarrow x_{n}$ as $i \rightarrow \infty$. Thus

$$
\left|\hat{F}_{n}^{(i)}(x)-\hat{F}_{n}(x)\right|<\frac{\delta}{3} \quad \text { for each } i>m_{0} .
$$

Since $\left(\hat{F}_{n}^{(j)}\right)$ is $I$-converges to $L_{j}$, there exists $D \in I$ such that, for each $n \notin D$, we have

$$
\left|\hat{F}_{n}^{(j)}(x)-L_{j}\right|<\frac{\delta}{3}
$$

Without loss of generality, let $j>m_{0}$. Then, for all $n \notin D$, we have by (2.13), (2.14), and (2.15) that

$$
\left|\hat{F}_{n}(x)-L\right| \leq\left|\hat{F}_{n}(x)-\hat{F}_{n}^{(j)}(x)\right|+\left|\hat{F}_{n}^{(j)}(x)-L_{j}\right|+\left|L_{j}-L\right|<\delta
$$


Hence $\left(x_{n}\right)$ is Fibonacci $I$-convergent to $L$. Thus $m^{I}(\hat{F})$ is a Banach space. The other cases can be similarly established.

The following results are consequences of Theorem 2.7.

Theorem 2.8 The spaces $m^{I}(\hat{F})$ and $m_{0}^{I}(\hat{F})$ are $K$-spaces.

Theorem 2.9 The set $m^{I}(\hat{F})$ is a closed subspace of $\ell_{\infty}(\hat{F})$.

Since the inclusions $m^{I}(\hat{F}) \subset \ell_{\infty}(\hat{F})$ and $m_{0}^{I}(\hat{F}) \subset \ell_{\infty}(\hat{F})$ are strict, in view of Theorem 2.9 , we have the following result.

Theorem 2.10 The spaces $m^{I}(\hat{F})$ and $m_{0}^{I}(\hat{F})$ are nowhere dense subsets of $\ell_{\infty}(\hat{F})$.

Theorem 2.11 The spaces $c_{0}^{I}(\hat{F})$ and $m_{0}^{I}(\hat{F})$ are solid and monotone.

Proof We will prove the result for $c_{0}^{I}(\hat{F})$; for $m_{0}^{I}(\hat{F})$, the result can be established similarly. Let $x=\left(x_{n}\right) \in c_{0}^{I}(\hat{F})$. For $\epsilon>0$, the set

$$
\left\{n \in \mathbb{N}:\left|\hat{F}_{n}(x)\right| \geq \epsilon\right\} \in I
$$

Let $\alpha=\left(\alpha_{n}\right)$ be a sequence of scalars with $|\alpha| \leq 1$ for all $n \in \mathbb{N}$. Then

$$
\left|\hat{F}_{n}(\alpha x)\right|=\left|\alpha \hat{F}_{n}(x)\right| \leq|\alpha|\left|\hat{F}_{n}(x)\right| \leq\left|\hat{F}_{n}(x)\right| \quad \text { for all } n \in \mathbb{N}
$$

From this inequality and from (2.16) we have that

$$
\left\{n \in \mathbb{N}:\left|\hat{F}_{n}(\alpha x)\right| \geq \epsilon\right\} \subseteq\left\{n \in \mathbb{N}:\left|\hat{F}_{n}(x)\right| \geq \epsilon\right\} \in I
$$

implies

$$
\left\{n \in \mathbb{N}:\left|\hat{F}_{n}(\alpha x)\right| \geq \epsilon\right\} \in I
$$

Therefore $\left(\alpha x_{n}\right) \in c_{0}^{I}(\hat{F})$. Hence the space $c_{0}^{I}(\hat{F})$ is solid, and hence by Lemma 1.1 the space $c_{0}^{I}(\hat{F})$ is monotone.

Theorem 2.12 The spaces $c_{0}^{I}(\hat{F})$ and $c^{I}(\hat{F})$ are sequence algebras.

Proof Let $x=\left(x_{n}\right), y=\left(y_{n}\right) \in c_{0}^{I}(\hat{F})$. Then

$$
I-\lim _{n \rightarrow \infty}\left|\hat{F}_{n}(x)\right|=0 \quad \text { and } \quad I-\lim _{n \rightarrow \infty}\left|\hat{F}_{n}(y)\right|=0 .
$$

Therefore, from (2.17) we have $I-\lim \left|\hat{F}_{n}(x \cdot y)\right|=0$. This implies that $\left\{n \in \mathbb{N}:\left|\hat{F}_{n}(x \cdot y)\right| \geq\right.$ $\epsilon\} \in I$. Thus, $(x \cdot y) \in c_{0}^{I}(\hat{F})$. Hence $c_{0}^{I}(\hat{F})$ is sequence algebra. Similarly, we can prove that $c^{I}(\hat{F})$, is a sequence algebra.

Theorem 2.13 The function $h: m^{I}(\hat{F}) \rightarrow \mathbb{R}$ defined by $h(x)=\left|I-\lim \hat{F}_{n}(x)\right|$, where $m^{I}(\hat{F})=$ $\ell_{\infty}(\hat{F}) \cap c^{I}(\hat{F})$, is a Lipschitz function and hence uniformly continuous. 
Proof First of all, we show that the function is well defined. Let $x, y \in m^{I}(\hat{F})$ be such that

$$
\begin{aligned}
x=y & \Rightarrow I-\lim \hat{F}_{n}(x)=I-\lim \hat{F}_{n}(y) \\
& \Rightarrow \quad\left|I-\lim \hat{F}_{n}(x)\right|=\left|I-\lim \hat{F}_{n}(y)\right| \quad \Rightarrow \quad h(x)=h(y) .
\end{aligned}
$$

Thus $h$ is well defined. Next, let $x=\left(x_{n}\right), y=\left(y_{n}\right) \in m^{I}(\hat{F}), x \neq y$. Then

$$
A_{x}=\left\{n \in \mathbb{N}:\left|\hat{F}_{n}(x)-h(x)\right| \geq|x-y|_{*}\right\} \in I
$$

and

$$
A_{y}=\left\{n \in \mathbb{N}:\left|\hat{F}_{n}(y)-h(y)\right| \geq|x-y|_{*}\right\} \in I,
$$

where $|x-y|_{*}=\sup _{n}\left|\hat{F}_{n}(x)-\hat{F}_{n}(y)\right|$. Thus

$$
B_{x}=\left\{n \in \mathbb{N}:\left|\hat{F}_{n}(x)-h(x)\right|<|x-y|_{*}\right\} \in \mathcal{F}(I)
$$

and

$$
B_{y}=\left\{n \in \mathbb{N}:\left|\hat{F}_{n}(y)-h(y)\right|<|x-y|_{*}\right\} \in \mathcal{F}(I) .
$$

Hence $B=B_{x} \cap B_{y} \in \mathcal{F}(I)$, so that $B$ is a nonempty set. Therefore, choosing $n \in B$, we have

$$
\begin{aligned}
|h(x)-h(y)| & \leq\left|h(x)-\hat{F}_{n}(x)\right|+\left|\hat{F}_{n}(x)-\hat{F}_{n}(y)\right|+\left|\hat{F}_{n}(y)-h(y)\right| \\
& \leq 3|x-y|_{*} .
\end{aligned}
$$

Thus, $h$ is a Lipschitz function and hence uniformly continuous.

Theorem 2.14 If $x=\left(x_{n}\right), y=\left(y_{n}\right) \in m^{I}(\hat{F})$ with $\hat{F}_{n}(x \cdot y)=\hat{F}_{n}(x) \cdot \hat{F}_{n}(y)$, then $(x \cdot y) \in m^{I}(\hat{F})$ and $h(x \cdot y)=h(x) \cdot h(y)$, where $h: m^{I}(\hat{F}) \rightarrow \mathbb{R}$ is defined by $h(x)=\left|I-\lim \hat{F}_{n}(x)\right|$.

Proof For $\epsilon>0$,

$$
B_{x}=\left\{n \in \mathbb{N}:\left|\hat{F}_{n}(x)-h(x)\right|<\epsilon\right\} \in \mathcal{F}(I)
$$

and

$$
B_{y}=\left\{n \in \mathbb{N}:\left|\hat{F}_{n}(y)-h(y)\right|<\epsilon\right\} \in \mathcal{F}(I),
$$

where $\epsilon=|x-y|_{*}=\sup _{n}\left|\hat{F}_{n}(x)-\hat{F}_{n}(y)\right|$. Now, we have

$$
\begin{aligned}
\left|\hat{F}_{n}(x \cdot y)-h(x) h(y)\right| & =\left|\hat{F}_{n}(x) \hat{F}_{n}(y)-\hat{F}_{n}(x) h(y)+\hat{F}_{n}(x) h(y)-h(x) h(y)\right| \\
& \leq\left|\hat{F}_{n}(x)\right|\left|\hat{F}_{n}(y)-h(y)\right|+|h(y)|\left|\hat{F}_{n}(x)-h(x)\right| .
\end{aligned}
$$


As $m^{I}(\hat{F}) \subseteq \ell_{\infty}(\hat{F})$, there exists $M \in \mathbb{R}$ such that $\left|\hat{F}_{n}(x)\right|<M$. Therefore, from equations (2.18), (2.19), and (2.20) we have

$$
\begin{aligned}
\left|\hat{F}_{n}(x y)-h(x) h(y)\right| & =\left|\hat{F}_{n}(x) \cdot \hat{F}_{n}(y)-h(x) h(y)\right| \\
& \leq M \epsilon+|h(y)| \epsilon=\epsilon_{1} \quad \text { (say) }
\end{aligned}
$$

for all $n \in B_{x} \cap B_{y} \in \mathcal{F}(I)$. Hence $(x \cdot y) \in m^{I}(\hat{F})$ and $h(x \cdot y)=h(x) \cdot h(y)$.

\title{
3 Conclusion
}

In this paper, we have introduced and studied new difference sequence spaces $c_{0}^{I}(\hat{F})$, $c^{I}(\hat{F})$, and $\ell_{\infty}^{I}(\hat{F})$. We investigated the general type of convergence, that is, Fibonacci $I-$ convergence for sequences related to the Fibonacci difference matrix $\hat{F}$ derived by the sequence of Fibonacci numbers. We studied some inclusion relations concerning these spaces. Further, we investigated some topological and algebraic properties of these spaces. These definitions and results provide new tools to deal with the convergence problems of sequences occurring in many branches of science and engineering.

\section{Acknowledgements}

The authors would like to thank the referees for a careful reading and several constructive comments and making some useful corrections that have improved the presentation of the paper.

\section{Funding}

This work was supported by Department of Mathematics, Amman Arab University, Amman, Jordan.

\section{Competing interests}

The authors declare that they have no competing interests.

\section{Authors' contributions}

All authors of the manuscript have read and agreed to its content and are accountable for all aspects of the accuracy and integrity of the manuscript.

\begin{abstract}
Authors' information
Vakeel A. Khan received the M.Phil. and Ph.D., degrees in Mathematics from Aligarh Muslim University, Aligarh, India. Currently, he is an Associate Professor at Aligarh Muslim University, Aligarh, India. A vigorous researcher in the area of sequence spaces, he has published a number of research papers in reputed national and international journals, including Numerical Functional Analysis and Optimization (Taylors and Francis), Information Sciences (Elsevier), Applied

Mathematics Letters (Elsevier), A Journal of Chinese Universities (Springer-Verlag, China). Rami K.A. Rababah is working as an Assistant Professor in the Department of Mathematics, Amman Arab University, Jordan. Kamal M.A.S. Alshlool received M.Sc., from Aligarh Muslim University and is currently a Ph.D., scholar at Aligarh Muslim University. Sameera A.A. Abdullah received M.Sc., from Aligarh Muslim University and is currently a Ph.D., scholar at Aligarh Muslim University. Ayaz Ahmad is working as an Assistant Professor in the National Institute Technology, Patna, India.
\end{abstract}

\section{Author details}

'Department of Mathematics, Aligarh Muslim University, Aligarh, India. ${ }^{2}$ Department of Mathematics, Amman Arab University, Amman, Jordan. ${ }^{3}$ Department of Mathematics, National Institute of Technology, Patna, India.

\section{Publisher's Note}

Springer Nature remains neutral with regard to jurisdictional claims in published maps and institutional affiliations.

Received: 23 January 2018 Accepted: 10 May 2018 Published online: 25 May 2018

\section{References}

1. Başarir, M., Başar, F., Kara, E.E.: On the spaces of Fibonacci difference absolutely $p$-summable, null and convergent sequences. Sarajevo J. Math. 12(25)(2), 167-182 (2016)

2. Candan, M.: Domain of the double sequential band matrix in the spaces of convergent and null sequences. Adv. Differ. Equ. 2014(1), 163 (2014)

3. Candan, M., Kara, E.E.: A study on topological and geometrical characteristics of new Banach sequence spaces. Gulf J. Math. 3(4), 67-84 (2015)

4. Candan, M., Kayaduman, K.: Almost convergent sequence space derived by generalized Fibonacci matrix and Fibonacci core. Br. J. Math. Comput. Sci. 7(2), 150-167 (2015) 
5. Das, A., Hazarika, B.: Some new Fibonacci difference spaces of non-absolute type and compact operators. Linear Multilinear Algebra 65(12), 2551-2573 (2017)

6. Das, A., Hazarika, B.: Matrix transformation of Fibonacci band matrix on generalized bv-space and its dual spaces. Bol. Soc. Parana. Mat. 36(3), 41-52 (2018)

7. Das, A., Hazarika, B., Kara, E.E., Başar, F.: On composition operators of Fibonacci matrix and applications of Hausdorff measure of noncompactness. Bol. Soc. Parana. Mat. (accepted)

8. Das, P., Kostyrko, P., Wilczynski, W., Malik, P.: / and /*-convergence of double sequences. Math. Slovaca 58(5), 605-620 (2008)

9. Fast, H.: Sur la convergence statistique. In: Colloquium Mathematicae, vol. 2, p. 241-244 (1951)

10. Hazarika, B., Das, A.: Some properties of generalized Fibonacci difference bounded and $p$-absolutely convergent sequences. Bol. Soc. Parana. Mat. 36(1), 37-50 (2018)

11. Hazarika, B., Tamanag, K.: On Zweier generalized difference ideal convergent sequences in a locally convex space defined by Musielak-Orlicz function. Bol. Soc. Parana. Mat. 35(2), 19-37 (2016)

12. Kara, E.E.: Some topological and geometrical properties of new Banach sequence spaces. J. Inequal. Appl. 2013(1), 38 (2013)

13. Kara, E.E., Başarir, M.: An application of Fibonacci numbers into infinite Toeplitz matrices. Casp. J. Math. Sci. 1(1), 43-47 (2012)

14. Kara, E.E., Daştan, M., Illkhan, M.: On almost ideal convergence with respect to an Orlicz function. Konuralp J. Math. 4(2), 87-94 (2016)

15. Kara, E.E., Ilkhan, M.: On some Banach sequence spaces derived by a new band matrix. Br. J. Math. Comput. Sci. 9(2), 141-159 (2015)

16. Kara, E.E., Ilkhan, M.: On some paranormed A-ideal convergent sequence spaces defined by Orlicz function. Asian J. Math. Comput. Res. 4(4), 183-194 (2015)

17. Kara, E.E., Illkhan, M.: Lacunary 1 -convergent and lacunary /-bounded sequence spaces defined by an Orlicz function. Electron. J. Math. Anal. Appl. 4(2), 150-159 (2016)

18. Kara, E.E., llkhan, M.: Some properties of generalized Fibonacci sequence spaces. Linear Multilinear Algebra 64(11), 2208-2223 (2016)

19. Khan, V.A., Ebadullah, K.: I-Convergent difference sequence spaces defined by a sequence of moduli. J. Math. Comput. Sci. 2(2), 265-273 (2012)

20. Kiriş̧̧, M.: The application domain of infinite matrices with algorithms. Univers. J. Math. Appl. 1(1), 1-9 (2018)

21. Kiriş̧̧i, M., Karaisa, A.: Fibonacci numbers, statistical convergence and applications. Preprint. arXiv:1607.02307 (2016)

22. Kizmaz, H.: Certain sequence spaces. Can. Math. Bull. 24(2), 169-176 (1981)

23. Koshy, T.: Fibonacci and Lucas Numbers with Applications. Wiley, New York (2001)

24. Kostyrko, P., Macaj, M., Šalát, T.: Statistical convergence and I-convergence. In: Real Analysis Exchange (1999)

25. Murat, K., Karaisa, A.: Fibonacci statistical convergence and Korovkin type approximation theorems. J. Inequal. Appl. $2017,229(2017)$

26. Mursaleen, M., Noman, A.K.: On some new sequence spaces of non-absolute type related to the spaces $\ell_{p}$ and $\ell_{\infty}$. Filomat 25(2), 33-51 (2011)

27. Šalát, T., Tripathy, B.C., Ziman, M.: On some properties of I-convergence. Tatra Mt. Math. Publ. 28(2), 274-286 (2004)

28. Šalát, T., Tripathy, B.C., Ziman, M.: On I-convergence field. Ital. J. Pure Appl. Math. 17(5), 1-8 (2005)

29. Steinhaus, H.: Sur la convergence ordinaire et la convergence asymptotique. In: Colloquium Mathematicae, vol. 2, pp. 73-74 (1951)

30. Tripathy, B., Hazarika, B.: Paranorm I-convergent sequence spaces. Math. Slovaca 59(4), 485-494 (2009)

31. Tripathy, B.C., Hazarika, B.: I-Convergent sequence spaces associated with multiplier sequences. Math. Inequal. Appl. $11(3), 543-548(2008)$

32. Tripathy, B.C., Hazarika, B.: Some I-convergent sequence spaces defined by Orlicz functions. Acta Math. Appl. Sin. Engl. Ser. 27(1), 149-154 (2011)

\section{Submit your manuscript to a SpringerOpen ${ }^{\circ}$ journal and benefit from:}

- Convenient online submission

- Rigorous peer review

- Open access: articles freely available online

- High visibility within the field

- Retaining the copyright to your article

Submit your next manuscript at $\gg$ springeropen.com 